\title{
Complementary Imaging of Silver Nanoparticle Interactions with Green Algae: Dark-Field Microscopy, Electron Microscopy, and Nanoscale Secondary Ion Mass Spectrometry
}

Ryo Sekine, ${ }^{*, \dagger, \ddagger, \# \odot ~ K a t i e ~ L . ~ M o o r e, ~}{ }^{\S, \|, \#}$ Marianne Matzke, ${ }^{\ddagger}$ Pascal Vallotton, ${ }^{\perp, \square}$ Haibo Jiang, ${ }^{\S, \text { I }}$ Gareth M. Hughes, ${ }^{\S}$ Jason K. Kirby, ${ }^{\text {II }}$ Erica Donner, ${ }^{\dagger}$ Chris R. M. Grovenor, ${ }^{\S}$ Claus Svendsen, ${ }^{\ddagger}$ and Enzo Lombi ${ }^{\dagger}$

${ }^{\dagger}$ Future Industries Institute, University of South Australia, Building X, Mawson Lakes Campus, Adelaide, SA 5095, Australia

${ }^{\ddagger}$ Centre for Ecology and Hydrology, Maclean Building, Benson Lane, Crowmarsh Gifford, Wallingford, Oxfordshire OX10 8BB, United Kingdom

${ }^{\S}$ Department of Materials, The University of Oxford, Parks Road, Oxford, OX1 3PH, United Kingdom

"School of Materials, The University of Manchester, Oxford Road, Manchester, M13 9PL, United Kingdom

${ }^{\perp}$ Institute für Biochemie, ETH Zurich, Otto-Stern-Weg 3, 8093 Zürich, Switzerland

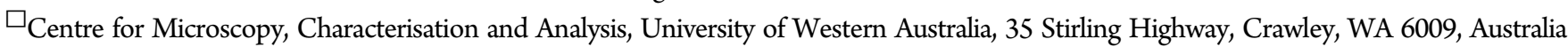

${ }^{\text {II } C S I R O ~ L a n d ~ a n d ~ W a t e r, ~ E n v i r o n m e n t a l ~ C o n t a m i n a n t ~ M i t i g a t i o n ~ a n d ~ B i o t e c h n o l o g y ~ P r o g r a m, ~ W a i t e ~ C a m p u s, ~ W a i t e ~ R o a d, ~}$

Perth, SA 5064, Australia

Supporting Information
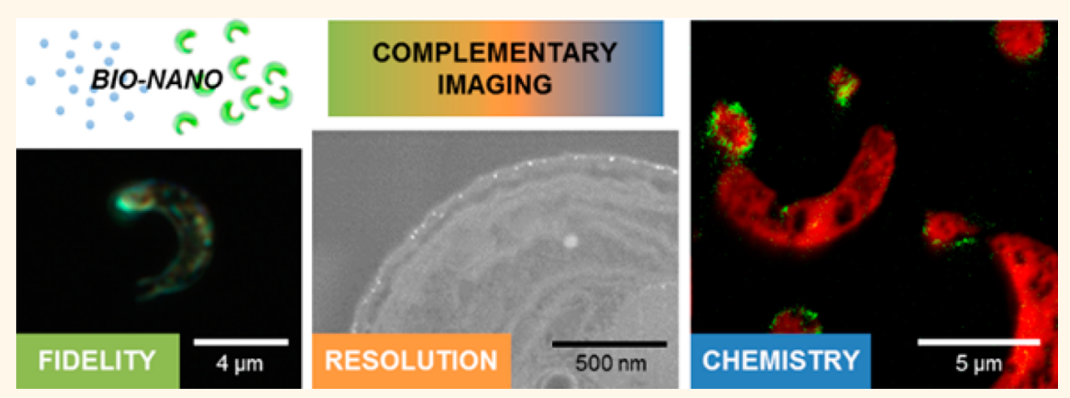

ABSTRACT: Increasing consumer use of engineered nanomaterials has led to significantly increased efforts to understand their potential impact on the environment and living organisms. Currently, no individual technique can provide all the necessary information such as their size, distribution, and chemistry in complex biological systems. Consequently, there is a need to develop complementary instrumental imaging approaches that provide enhanced understanding of these "bio-nano" interactions to overcome the limitations of individual techniques. Here we used a multimodal imaging approach incorporating dark-field light microscopy, high-resolution electron microscopy, and nanoscale secondary ion mass spectrometry (NanoSIMS). The aim was to gain insight into the bio-nano interactions of surface-functionalized silver nanoparticles (Ag-NPs) with the green algae Raphidocelis subcapitata, by combining the fidelity, spatial resolution, and elemental identification offered by the three techniques, respectively. Each technique revealed that Ag-NPs interact with the green algae with a dependence on the size $(10 \mathrm{~nm} v s 60 \mathrm{~nm}$ ) and surface functionality (tannic acid vs branched polyethylenimine, bPEI) of the NPs. Dark-field light microscopy revealed the presence of strong light scatterers on the algal cell surface, and SEM imaging confirmed their nanoparticulate nature and localization at nanoscale resolution.

continued...

Received: June 29, 2017

Accepted: October 23, 2017

Published: October 23, 2017 
NanoSIMS imaging confirmed their chemical identity as $\mathrm{Ag}$, with the majority of signal concentrated at the cell surface. Furthermore, SEM and NanoSIMS provided evidence of $10 \mathrm{~nm}$ bPEI Ag-NP internalization at higher concentrations $(40 \mu \mathrm{g} / \mathrm{L})$, correlating with the highest toxicity observed from these NPs. This multimodal approach thus demonstrated an effective approach to complement dose-response studies in nano-(eco)-toxicological investigations.

KEYWORDS: $\quad$ silver nanoparticles, multimodal imaging, NanoSIMS, dark-field light microscopy, bio-nano interactions, nanotoxicology

$\mathrm{N}$ anotechnology-the set of technologies associated with engineered materials at the nanometer scale-has revolutionized many industries. It has seen tremendous advances over the last few decades and is now used in a diverse range of products (see examples in the Nanodatabase and the Project for Emerging Technologies). ${ }^{1,2}$ These applications exploit the unique geometric and/or quantum properties that arise when materials are manufactured at the extremely small scale referred to as "nanoscale", such as their high specific surface area (SSA) or optical resonances. Importantly, it is now recognized that, due to their small size and tailored surface chemistries, engineered nanomaterials (ENMs) may interact in a distinct manner with living organisms in the environment compared to their dissolved or macroscopic counterparts. ${ }^{3}$ However, instrumental techniques capable of elucidating the interactions of ENMs with living systems (i.e., bio-nano interactions) in complex biological matrices are often technically difficult, expensive, and time-consuming to perform. ${ }^{4}$ This is a serious research and regulatory challenge given the inevitability of ENMs being released into the environment as a consequence of the development and use of ENM-containing products. ${ }^{5,6}$

One set of instrumental approaches that is effective for investigating bio-nano interactions is imaging. An image that directly captures ENM interactions with organisms can contribute crucial information to advance the mechanistic understanding of their ecotoxicological impact, for example, by determining the site of ENM interaction and/or internalization. There are a number of imaging techniques available that exploit light or electrons (e.g., transmission imaging, fluorescence microscopy), surface topography (e.g., scanning probe microscopy), or chemistry (e.g., laser ablation ICP-MS), but each have their strengths and limitations. For example, conventional light microscopy can often be performed rapidly with minimal sample preparation, and it is therefore ideal for capturing live systems or delicate interactions without disruptive treatments. However, resolution is limited by diffraction (for visible light, $d \approx \lambda / 2 \approx$ $250 \mathrm{~nm}$ ) so that objects that are less than $d$ apart cannot be distinguished from one another. This is inadequate to resolve ENMs, whose sizes, by definition, are less than $100 \mathrm{~nm}$ in at least one dimension. ${ }^{7}$ This limitation can be overcome by using electron microscopy (EM) techniques: both scanning and transmission electron microscopies (SEM and TEM) are capable of providing visual identification of ENMs at the nanoscale, and they are the most widely used methods for imaging bio-nano interactions. Moreover, while EM has traditionally required fixed, dry samples, the development of environmental (low-vacuum) $\mathrm{SEM}^{8,9}$ or cryo-EM ${ }^{10}$ has enabled high-resolution imaging of samples under "hydrated" conditions.

However, despite these technological advances, elemental identification at the nanoscale is still challenging. While EM systems equipped with energy-dispersive X-ray spectrometers (EDX) offer both visual and elemental identification at sub-micrometer scales, there are limitations in volumetric resolution. SEM-EDX often suffers from large subsurface interaction volumes (and hence decreased lateral resolution), and while
TEM circumvents this by virtue of thin sections, the depth resolution is effectively limited by the section thickness. Synchrotron-based X-ray fluorescence microscopy (XFM) techniques are now capable of attaining images at single-ENMlevel resolution with chemical information; ${ }^{11,12}$ however, only few leading facilities are capable of this performance and accessibility is limited. Indeed, there is no single independent technique that can simultaneously provide nanoscale spatial resolution and nanoscale chemical information without some loss of sample fidelity. Thus, complementary approaches are needed that combine techniques with strengths in imaging and elemental characterization to overcome the individual limitations and provide a comprehensive understanding of ENM biointeractions.

In this study, we address this analytical challenge by employing a combination of three complementary imaging techniques to investigate the bio-nano interactions between silver nanoparticles (Ag-NPs) and the algae Raphidocelis subcapitata (formerly known as Pseudokirchneriella subcapitata and Selenastrum capricornutum). Of the thousands of ENMs that have been identified in the consumer market, Ag-NPs dominate in terms of product range and availability and hence are one of the most likely ENMs to enter the environment. ${ }^{13}$ Furthermore, ionic silver $\left(\mathrm{Ag}^{+}\right)$is known to be toxic toward a variety of organisms in aquatic and terrestial enviornments. For this reason, Ag-NPs have been of particular focus in many studies. ${ }^{14-17} R$. subcapitata is a unicellular, autotrophic algae specified by the OECD as a model species in its guidelines for freshwater toxicity testing ${ }^{18}$ and is therefore an important, widely adoped organism in environmental nanotoxicology. ${ }^{14,19,20}$ By using a combination of light-, electron-, and mass-based microscopies, we demonstrate an effective approach to visualize and chemically identify Ag-NPs and their interactions with this model organism.

Specifically, we identified Ag-NPs interacting with $R$. subcapitata by dark-field light microscopy (DF-LM) under aqueous conditions, localized them with high resolution using SEM and confirmed the chemical identity by using nanoscale secondary ion mass spectrometry (NanoSIMS) imaging. Dark-field light microscopy, which captures scattered light from the sample, is ideal for identifying strongly scattering objects such as Ag-NPs in lowscattering matrices (e.g., water or cells). Recent developments have enabled successful identification and quantification of different ENMs in aqueous dispersions ${ }^{21}$ and imaging of ENMs' interaction with single cells ${ }^{22,23}$ and even with larger model organisms. ${ }^{24}$ Importantly, this can be performed under aqueous conditions with minimal sample preparation, thus maximizing the fidelity of the potentially delicate interactions. NanoSIMS is an ion nanoprobe that simultaneously collects multiple secondary ions generated from the sample surface to create elemental maps at high lateral resolution $(50 \mathrm{~nm})$ and with high surface specificity (a few atomic layers). ${ }^{25}$ Combined with highresolution SEM, the NanoSIMS technique has been exploited for its unrivalled nanoscale chemical sensitivity to analyze metal and micronutrient $(\mathrm{mg} / \mathrm{kg}$ concentrations or less) distributions in, for example, soils and interfaces ${ }^{26-28}$ and plants, ${ }^{13,29,30}$ and 
Table 1. Complementarity of the Dark-Field Light Microscopy (DF-LM), Electron Microscopy (SEM and TEM), and Nanoscale Secondary Ion Mass Spectrometry (NanoSIMS) at a Glance with Strengths Graded from Low $(+)$ to High $(+++)$

$\begin{array}{lccccc}\text { fidelity } & \text { resolution } & \text { chemistry } & \text { limitations } \\ \text { DF-LM } & +++ & + & ++ & \begin{array}{c}\text { no/minimal sample preparation; ease of } \\ \text { operation }\end{array} & \text { limited spatial resolution; limited particle identification } \\ \text { NanoSIMS } & + & +++ & ++ & \begin{array}{c}\text { high spatial resolution }(\ll 10 \mathrm{~nm}) ; \\ \text { commonly available } \\ \text { nonoscale chemistry }(\geq 50 \mathrm{~nm}) ; \text { high } \\ \text { sensitivity }\end{array} & \begin{array}{c}\text { moderate to complex sample preparation; limited nanoscale chemistry; } \\ \text { vacuum required } \\ \text { complex sample preparation; difficult to differentiate NP from localized } \\ \text { ions; ultrahigh vacuum required }\end{array}\end{array}$
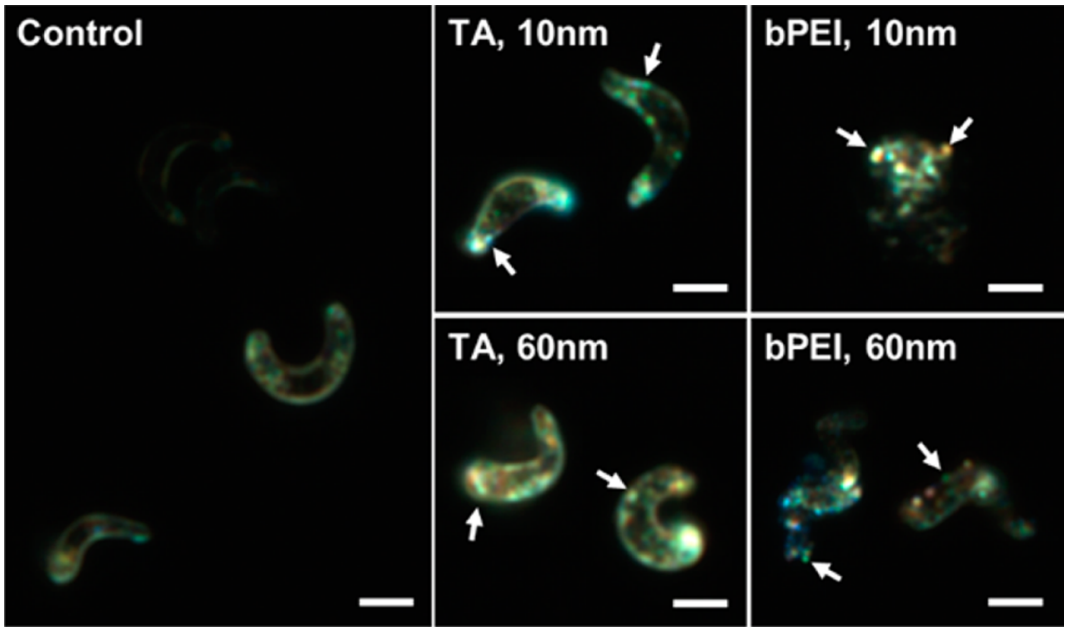

Figure 1. Dark-field microscopy images of R. subcapitata exposed to TA- and bPEI-Ag-NPs at $40 \mu \mathrm{g} / \mathrm{L}$. TA-Ag-NPs do not affect the cell morphology significantly and show brighter scattering along the cell walls beyond that of the controls. bPEI-Ag-NPs-exposed cells show strong scattering features, but cell shapes are severely affected, particularly for the $10 \mathrm{~nm}$ Ag-NPs. Examples of point scatterers that most likely represent Ag-NPs are indicated by arrows. Scale bar $=2 \mu \mathrm{m}$.

has recently been applied to study Ag-NPs' interactions with bacteria, ${ }^{31}$ algae, ${ }^{31,32}$ and the crustacean Daphnia magna. ${ }^{31}$ However, since the analysis is performed under ultrahigh vacuum $\left(10^{-10}\right.$ Torr $)$, water must be removed from biological samples, and these must be appropriately fixed, embedded, and sectioned, which may alter the sample fidelity. Therefore, DF-LM was necessary to complete the methodology by confirming the sample preparation steps had not altered the distribution. Here, we demonstrate the effectiveness of this three-level multimodal approach that exploits their complementary strengths in sample fidelity (DF-LM), high spatial resolution (SEM), and nanoscale chemistry (NanoSIMS) for visually determining the localization of NPs in single-cell organisms (Table 1).

\section{RESULTS AND DISCUSSION}

Dark-Field Light Microscopy: Effect of Ag-NPs on the Growth of $R$. subcapitata. Exposure of $R$. subcapitata cultures to Ag-NPs caused size- and surface-dependent responses in the algae growth rate. For a given surface functionality, the smaller $10 \mathrm{~nm}$ Ag-NPs were more toxic than their $60 \mathrm{~nm}$ counterparts, while the positively charged branched polyethylenimine (bPEI) coating imparted greater toxicity than the negatively charged tannic acid (TA) coatings (Figure S1). These observations are in agreement with previously published findings, where smaller $\mathrm{Ag}$-NPs and positively charged bPEI-Ag-NPs were consistently reported to be more toxic based on equivalent $\mathrm{Ag}$ mass concentrations. ${ }^{14,33}$ The acquired DF-LM images reflect this, with the $10 \mathrm{~nm}$ bPEI-Ag-NPs at $40 \mu \mathrm{g} / \mathrm{L}$ exposure concentration severely affecting the algal cell morphology, so much so that only a few intact cells were observed. Control samples show a relatively dark and uniform image of the algal cells, while exposed cells scatter light more intensely and, as a result, they appear brighter in the dark-field images (Figure 1). Furthermore, the exposed cells appear to be brighter along the cell walls compared to the control, and there are multiple point scatterers most likely associated with Ag-NPs. The hyperspectral profiles of these spots show intense scattering at shorter wavelengths, which is typical of Ag-NPs (Figure S2) ${ }^{34}$ However, as this can change in response to dissolution (i.e., size) and their surface interactions in the host matrix, the profile alone is not sufficient to determine their identities. For example, the matrix-acquired coatings may have a different refractive index from the original coating or may induce plasmon resonance shifts to significantly alter the dark-field hyperspectral profiles. In a recent study, Théoret and Wilkinson noted the complexity of Ag-NP identification in wastewaters and suggested that the red-shift in the dark-field hyperspectral profile from Ag-NPs exposed to wastewater was due to the adsorption of organic matter. ${ }^{35}$ Other transformations such as dissolution ${ }^{36}$ (size change) or sulfidation (no plasmon resonance) of Ag-NPs would also affect their profiles. Finally, even though tomography can be used to determine attached $v$ s internalized NPs, ${ }^{14,37}$ the determination of their precise locations (e.g., immediately inside the cell or outside of the cell wall, on top of or underneath the cell, or in relation to any of its internal structures) is ultimately limited in spatial resolution by diffraction (ca. $250 \mathrm{~nm}$ ).

Nevertheless, within these limitations, DF-LM is a fast and effective technique to screen for potential bio-nano interactions under environmentally realistic aqueous conditions, as also demonstrated in other recent studies. ${ }^{14,22}$ While quantitative investigations were beyond the scope of this study, the rapid screening capability of DF-LM can offer such information in a time-resolved manner that is not practically feasible with SEM or 
NanoSIMS. For example, changes in cell volume and reproductive patterns have been reported recently in cells exposed to inorganic $^{38,39}$ and organic ${ }^{39}$ toxicant, that utilized phase contrast or differential interference microscopy combined with epifluorescence microscopy. Similarly, DF-LM could also be combined with fluorescent microscopy to provide correlative information regarding the bio-nano interactions that may be linked to morphological or behavioral changes, if such changes occur, in their life cycle.

Combined SEM and NanoSIMS Analyses: Control and $\mathrm{AgNO}_{3}$ Treatments. The SEM images of $\mathrm{R}$ subcapitata in control (no Ag exposure) and $\mathrm{AgNO}_{3}\left(2 \mu \mathrm{g} \mathrm{Ag}^{+} / \mathrm{L}\right.$, as dissolved Ag comparison) showed detailed cell structures with many of the organelles clearly distinguishable in backscattered electron (BSE) contrast (Figure 2). The cells also display some secondary electron (SE) contrast (Figure S3). The cell membranes and the lipid bodies appear brighter in the images, as do the starch granules and phosphate bodies. The nucleus is not clearly visible in this image, but the chloroplasts can be seen throughout the cells. There are some smaller particle-like features that are also electron dense that may be part of a metal-sequestering process. ${ }^{40}$ Both the control and $\mathrm{AgNO}_{3}$-exposed cells show these bright particles of varying sizes (ca. 20-100 nm), as indicated by the arrows in the BSE image of the unexposed algae (Figure 2). It should be noted here that many of these bright features are visible under SE contrast, but they do not always correlate to a positive contrast in BSE mode.

The NanoSIMS data set from $R$. subcapitata cells exposed to $2 \mu \mathrm{g} / \mathrm{L}$ of $\mathrm{AgNO}_{3}$ can be found in Figure 2, consisting of five images from the five secondary ion detectors ${ }^{12} \mathrm{C}^{14} \mathrm{~N}^{-},{ }^{32} \mathrm{~S}^{-}$, ${ }^{31} \mathrm{P}^{12} \mathrm{C}^{-},{ }^{107} \mathrm{Ag}^{-}$, and ${ }^{98} \mathrm{Mo}^{16} \mathrm{O}^{-}$distribution, as well as the SE detector image. The ${ }^{12} \mathrm{C}^{14} \mathrm{~N}^{-}$distribution map, commonly used as an indicator for proteins and/or biomass (cell morphology), clearly shows that the cells have retained their shape after fixation and is consistent with the SEM images (Figure S3). Both the control (Figure S4) and $\mathrm{AgNO}_{3}$-exposed algae show some $\mathrm{Ag}$ signal throughout the cells possibly due to interference from the ${ }^{95} \mathrm{Mo}^{12} \mathrm{C}^{-}$ion. Nevertheless, two key conclusions can be drawn from this data set regarding Ag analysis: (i) ${ }^{98} \mathrm{Mo}^{16} \mathrm{O}^{-}$signals are evenly distributed in the cells at very low intensities, and (ii) ${ }^{107} \mathrm{Ag}^{-}$has some areas of higher intensity but shows no greater correlation than ${ }^{98} \mathrm{Mo}^{16} \mathrm{O}^{-}$to the cell shape. Importantly, neither of the maps $\left({ }^{107} \mathrm{Ag}^{-}\right.$and $\left.{ }^{98} \mathrm{Mo}^{16} \mathrm{O}^{-}\right)$show regions of significant high intensity or "hot-spots" inside cells that could otherwise be attributable to the presence of Ag-NPs.

Combined SEM and NanoSIMS Analyses: Ag-NP Treatments. Due to their large backscatter yield, Ag-NPs were expected to appear as bright spots in the BSE images (due to high $\mathrm{Z}$ ) and also in SE images, as they may often introduce surface roughness to the sectioned surface. The $10 \mathrm{~nm}$ Ag-NPs were challenging to visualize using the SEM due to their small size and the minor but not insignificant charging of the resin material. The best images were acquired by minimizing the charging and maximizing surface contrast with low accelerating voltage $(1 \mathrm{kV})$, using the InLens SE detector equipped in the GEMINI column, which has a higher collection efficiency at short working distance. Figure 3 shows cross sections of algal cells exposed to $40 \mu \mathrm{g} / \mathrm{L}$ of $10 \mathrm{~nm}$ TA-Ag-NPs imaged at $5 \mathrm{kV}$ and at $1 \mathrm{kV}$, using the same InLens detector at a working distance (WD) of $3.4 \mathrm{~mm}$. The surface structural detail is blurred in the $5 \mathrm{kV}$ image, but bright particles are clearly visible on the algal cell wall in the $1 \mathrm{kV}$ image. Gaussian curve fitting of the line profiles

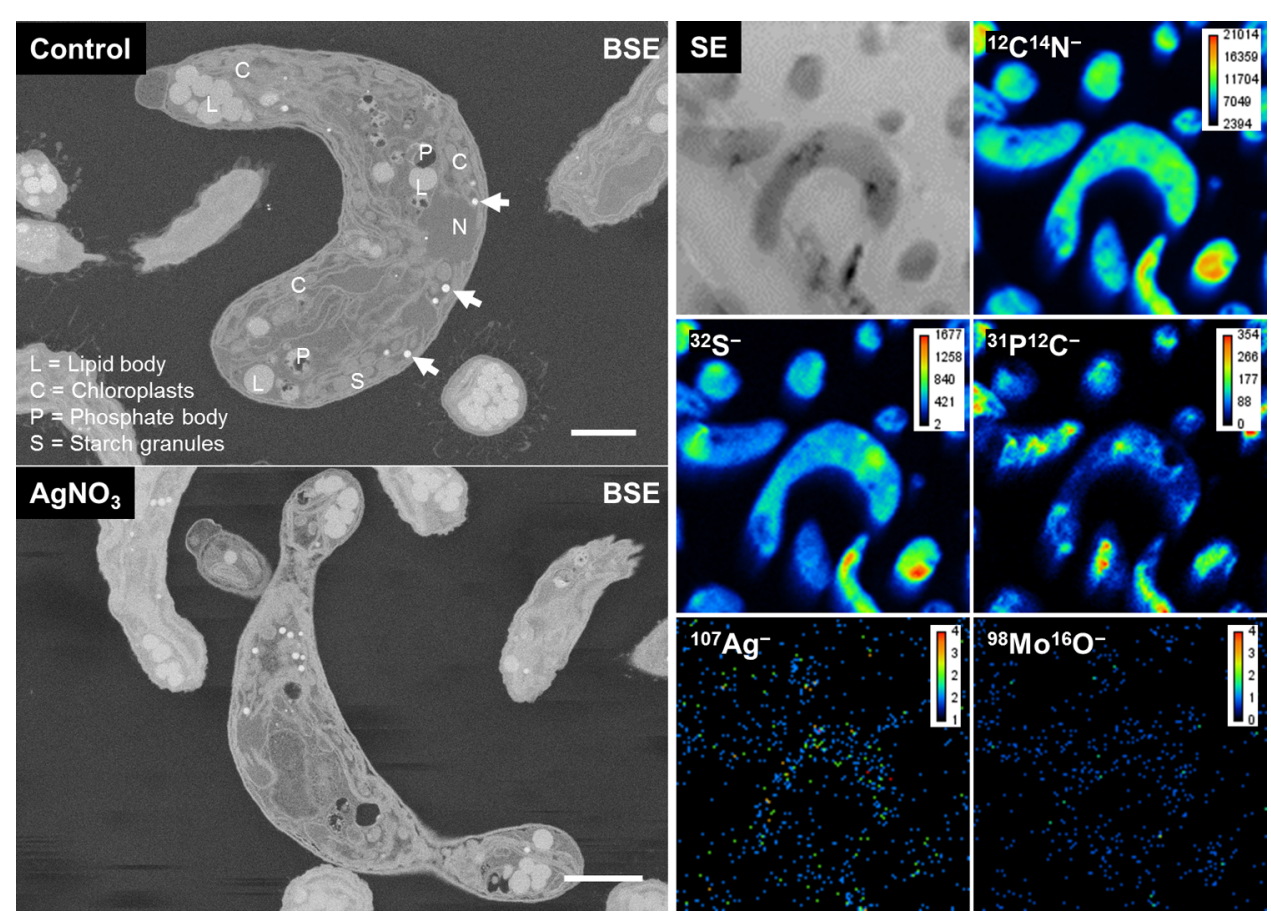

Figure 2. (Left) SEM images (BSE contrast) of control and $\mathrm{AgNO}_{3}$-exposed $\mathrm{R}$. subcapitata cells, with the organelles in the control exposure labeled as shown. Arrows indicate bright intracellular particles that were found in all cells including in the control samples. Scale bar $=1 \mu \mathrm{m}$. (Right) NanoSIMS ion images of $R$. subcapitata exposed to $2 \mu \mathrm{g} / \mathrm{L} \mathrm{Ag}^{+}$as $\mathrm{AgNO}_{3}$, showing the distributions of ${ }^{12} \mathrm{C}^{14} \mathrm{~N}^{-}$(indicative of cell morphology), ${ }^{32} \mathrm{~S}^{-}$and ${ }^{31} \mathrm{P}^{12} \mathrm{C}^{-}$(for phosphorus), ${ }^{107} \mathrm{Ag}^{-}$and ${ }^{98} \mathrm{Mo}^{16} \mathrm{O}^{-}$(for molybdenum), and the secondary electron (SE) image. ${ }^{98} \mathrm{Mo}^{16} \mathrm{O}^{-}$ has no mass interference with silver and shows low and uniform distribution in the cells. Similarly the ${ }^{107} \mathrm{Ag}^{-}$signal is low and uniform with no regions of significantly high $\mathrm{Ag}$ accumulation. The width of each NanoSIMS image is $15 \mu \mathrm{m}$. 

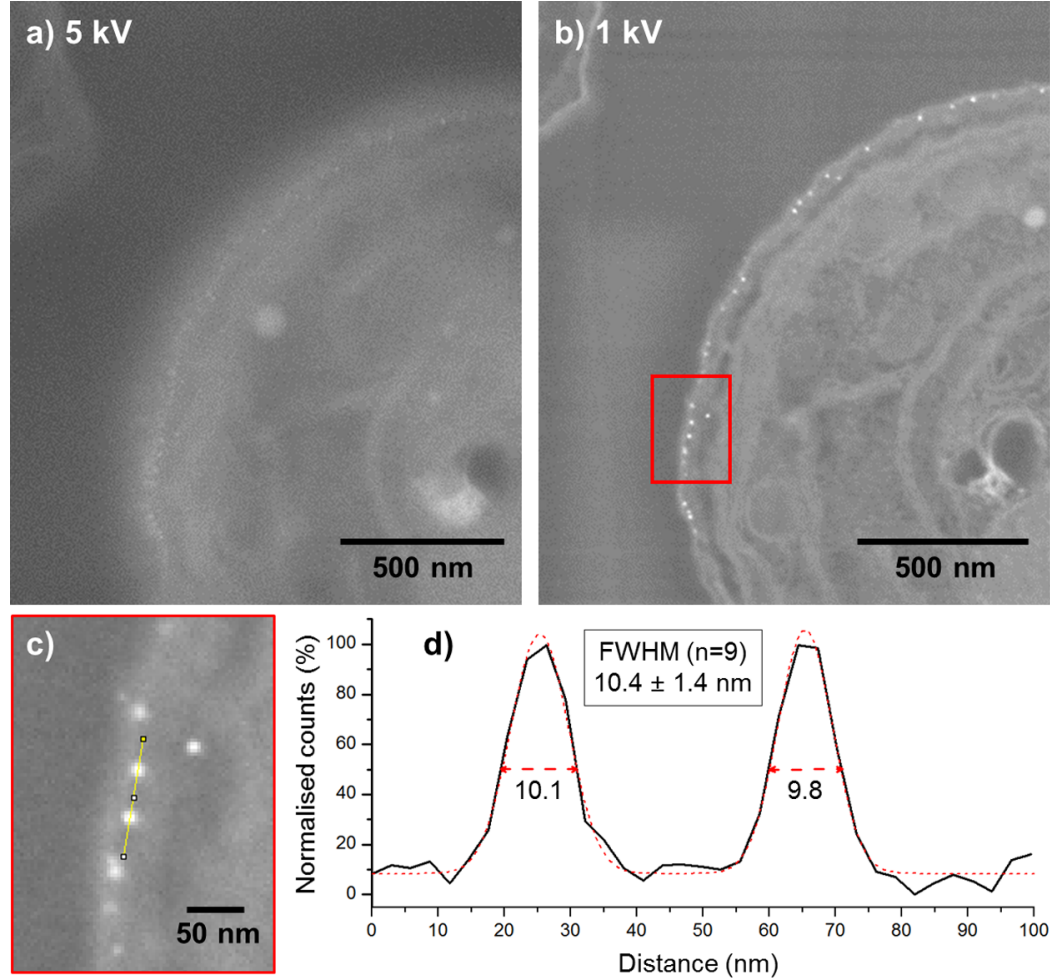

Figure 3. SE images of R. subcapitata exposed to $40 \mu \mathrm{g} / \mathrm{L}$ of $10 \mathrm{~nm}$ TA-Ag-NPs, imaged with (a) $5 \mathrm{kV}$ and (b) $1 \mathrm{kV}$ acceleration voltage (InLens $\mathrm{SE}, \mathrm{WD}=3.4 \mathrm{~mm})$. (c) Magnified image of the indicated area in (b) shows the line over which the profile (d) was generated. Red dotted curve shows the Gaussian profiles fitted to the experimental profile (solid black line) with a fwhm of 10.1 and $9.8 \mathrm{~nm}$. The average fwhm width of nine particles is $10.4 \pm 1.4 \mathrm{~nm}$, in agreement with the size of the Ag-NPs.

across nine individual particles confirms their fwhm size as $10.4 \pm 1.4 \mathrm{~nm}$, in agreement with the TEM size of the $10 \mathrm{~nm}$ TA-Ag-NPs. In all images of cells exposed to TA-Ag-NPs no conclusive evidence of Ag-NP internalization was observed.

Identification of the larger $60 \mathrm{~nm}$ Ag-NPs was unexpectedly compromised by the bright organelles of similar size within the cells (as shown in Figure 2), and it was therefore not possible to identify $60 \mathrm{~nm}$ Ag-NPs using BSE without additional chemical information. SEM-based EDX does not have adequate lateral resolution due to the large interaction volume; hence the NanoSIMS data are crucial to solving this challenge.

The NanoSIMS composite images of ${ }^{12} \mathrm{C}^{14} \mathrm{~N}^{-}$and ${ }^{107} \mathrm{Ag}^{-}$ion maps from algae exposed to $40 \mu \mathrm{g} / \mathrm{L}$ of $\mathrm{Ag}-\mathrm{NPs}$ can be found in Figure 4. Compared to the control (Figure S4) and $\mathrm{AgNO}_{3}$ (Figure 2) treatments, the ${ }^{107} \mathrm{Ag}^{-}$maps show $\mathrm{Ag}$ correlated with the cells $\left({ }^{12} \mathrm{C}^{14} \mathrm{~N}^{-}\right)$in a distinctly different manner. Both 10 and $60 \mathrm{~nm}$ TA-Ag-NPs show a similar mode of interaction with R. subcapitata, where ${ }^{107} \mathrm{Ag}^{-}$signals are detected around the perimeter of the cells defined by the ${ }^{12} \mathrm{C}^{14} \mathrm{~N}^{-}$ions for cells $1,3,4$, and 5 (10 nm TA-Ag-NPs) and cells 6-8 (60 nm TA-Ag-NPs). Other than the slight overlap of ${ }^{107} \mathrm{Ag}^{-}$with the ${ }^{12} \mathrm{C}^{14} \mathrm{~N}^{-}$signal observed in cell 2, there is no Ag detected inside cells. At the lower Ag-NP dose of $15 \mu \mathrm{g} / \mathrm{L}$ (data not shown), low ${ }^{107} \mathrm{Ag}^{-}$ signals were detected from algae using NanoSIMS. In these cells, the algal cells exposed to $10 \mathrm{~nm}$ Ag-NPs of both functionalities show some ${ }^{107} \mathrm{Ag}^{-}$. It is not easily distinguishable from the background levels in the control caused by interfering signals.

Similarly, the NanoSIMS images for $60 \mathrm{~nm}$ TA-Ag-NPs also suggest the adsorption of Ag-NPs on the surface of the algal cells. However, contrary to the $10 \mathrm{~nm}$ case, we were unable to identify $60 \mathrm{~nm}$ Ag-NPs near the cell walls in the SEM images. There are several possible explanations. First, it should be noted that only a few $60 \mathrm{~nm}$ TA-Ag-NPs were observed with each algae even with DF-LM, where the depth of field is as much as $0.5 \mu \mathrm{m}$ (much more than in SEM). Therefore, from a statistical perspective, it is likely that Ag-NPs are not present in the particular two-dimensional slice imaged with SEM, or there were too few Ag-NPs in the examined slice to be distinguished from the other particles in the cell. This is less likely to be an issue with the $10 \mathrm{~nm}$ Ag-NPs since the estimated Ag-NPs to algae molar ratio is in excess of 6000 to 1 as opposed to ca. 75 to 1 for $60 \mathrm{~nm} \mathrm{Ag-NPs.}$ However, NanoSIMS would also suffer from the same issue, so this is unlikely to be the only factor. The other possibility is partial dissolution of the NPs associated with the cells. Considering that the cell walls were brighter in the DF-LM images even in areas where Ag-NPs were not clearly observed, it is possible that this elevated scattering is produced by partially dissolved Ag-NPs associated with the cell membrane which were too small to be seen individually by SEM but large enough to increase the light-scattering intensity. Dissolved Ag will be difficult to visualize in the SEM especially in secondary electron mode unless $\mathrm{Ag}$ has reprecipitated and formed new (nano)structures. On the other hand, NanoSIMS will detect $\mathrm{Ag}$ regardless of its initial physicochemical form, provided that there is ${ }^{107} \mathrm{Ag}^{-}$generated in the sputtering volume. Such dissolution processes may be mediated by specific proteins or carbohydrates secreted by the cells in response to the presence of Ag-NPs, or it may have occurred during the sample preparation process for SEM and NanoSIMS.

In comparison, SEM images of cells exposed to $40 \mu \mathrm{g} / \mathrm{L}$ of $10 \mathrm{~nm}$ bPEI-Ag-NPs show bright particulate features that are inside the cells (Figure S5) that suggest that these NPs were internalized. The images show the bright NPs localized along the intracellular membranes. However, the estimated size of the NPs 
a) TA Ag-NPs $10 \mathrm{~nm}$

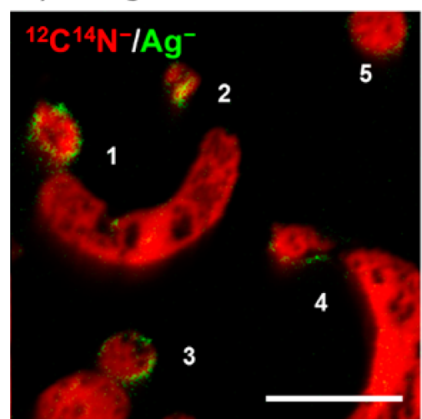

c) bPEI Ag-NPs $10 \mathrm{~nm}$

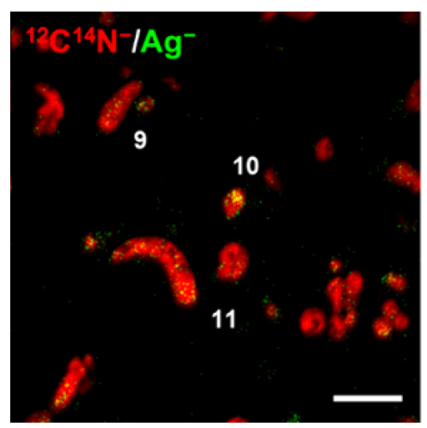

b) TA Ag-NPs $60 \mathrm{~nm}$

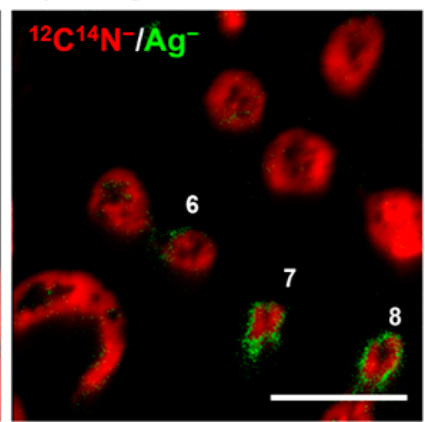

d) bPEI Ag-NPs $60 \mathrm{~nm}$

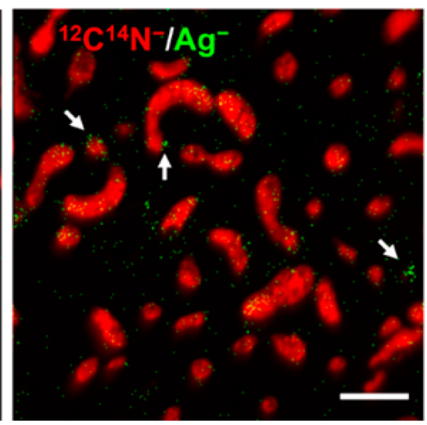

Figure 4. ${ }^{12} \mathrm{C}^{14} \mathrm{~N}^{-}$(red) and ${ }^{107} \mathrm{Ag}^{-}$(green) NanoSIMS composite images of $R$. subcapitata exposed to Ag-NPs of (a) $10 \mathrm{~nm}$ tannic acid (TA)-Ag-NPs, (b) $60 \mathrm{~nm}$ TA-Ag-NPs, (c) $10 \mathrm{~nm}$ polyethyleimine (bPEI)-Ag-NPs, and (d) $60 \mathrm{~nm}$ bPEI-Ag-NPs. Bright Ag fringes are particularly evident in TA-Ag-NPs exposed cells, while some $\mathrm{Ag}$ is also detected from inside the cells with $10 \mathrm{~nm}$ bPEI-Ag-NPs (cells 10 and 11). Scale bar indicates $5 \mu \mathrm{m}$.

is slightly larger than the expected size, with the Gaussian fwhm of $17.2 \pm 2.2 \mathrm{~nm}$ (average of $6 \mathrm{NPs}$ ) with some as large as $24.0 \mathrm{~nm}$ fwhm. In support of Ag accumulation, NanoSIMS images show Ag "hot-spots" observed inside some cells (e.g., cells 9-11) and suggest that the small particles observed in the SEM images may indeed be Ag-NPs (Figure S5). Only a very weak ${ }^{107} \mathrm{Ag}^{-}$signal was detected from cells exposed to $60 \mathrm{~nm}$ bPEI-Ag-NPs; however, the higher signals colocalized with higher ${ }^{12} \mathrm{C}^{14} \mathrm{~N}^{-}$(see arrows in Figure 4d), which also indicates some association with cells.

Interestingly, these SEM images and the ${ }^{12} \mathrm{C}^{14} \mathrm{~N}^{-}$map from NanoSIMS show more intact populations of cells compared to the DF-LM images (Figure 1), and the ${ }^{107} \mathrm{Ag}^{-}$distribution is not as well aligned to the cell structures as were the TA-Ag-NPs. Furthermore, the ${ }^{107} \mathrm{Ag}^{-}$signal appears less intense for bPEI-Ag-NPs compared to the TA-Ag-NPs, suggesting that there is an overall lower Ag content from the sampling area. This is surprising given that bPEI-Ag-NPs have a high attachment efficiency $(\alpha)$ and affinity for these cells. ${ }^{14}$ Similarly, positively charged allylamine coatings have been associated with strong attachment of gold nanoparticles to algae. ${ }^{41}$ One explanation may be their stability in the presence of exudates: the surface coating can influence the particle stability and behavior in the presence of algal exudates, and bPEI has been observed to be unstable in previous reports. ${ }^{14}$ It may also be that as some cells have been severely affected, Ag is associated more with the lysed cell contents and has not been retained through the sample preparation procedure. This highlights the importance of DF-LM in this multimodal approach, as a means to bring awareness to the potential effects of rigorous preparative treatments.

The images collectively demonstrate distinct interactions between $R$. subcapitata and Ag-NPs of both negative (TA) and positive (bPEI) surface functionalities. The type and extent of these interactions appear to be dependent on the particle size and surface functionality as expected from their different toxicity levels. For $10 \mathrm{~nm}$ TA-Ag-NPs, the images acquired from DF-LM (Figure 1), high-resolution SEM (Figure 3), and NanoSIMS (Figure 4) analyses collectively provide convincing evidence for the accumulation of Ag-NPs on the cell wall. The accumulation of $\mathrm{Ag}$ in the cell wall of algae has been reported previously for Chlamydomonas reinhardtii with PVP-Ag-NPs, ${ }^{32}$ and a similar observation was made with Euglena gracilis, where the Ag-NPs were sorbed to the pellicle (no cell wall). ${ }^{42}$

Interestingly, Wang et al. ${ }^{32}$ observed Ag inside the cytoplasm as sulfur-containing particles after uptake of the dissolved $\mathrm{Ag}^{+}$ into the cells, in addition to PVP-Ag-NPs accumulation in the periplasmic space. Similarly, accumulation of dissolved $\mathrm{Ag}$ and crystallization into $10 \mathrm{~nm} \mathrm{Ag-NPs}$ have also been reported previously. ${ }^{43}$ While our NanoSIMS data do not necessary correlate Ag with areas of high sulfur, the particles in the SEM correspond in size to the reported deposits in Ag-exposed C. reinhardtii and can be seen located along the intracellular membranes (Figure S4). These observations support our SEM and NanoSIMS data that suggest Ag presence in algae exposed to $10 \mathrm{~nm}$ bPEI-Ag-NPs with accumulation beyond the periplasmic space. On the other hand, we did not observe conclusive evidence for Ag inside the cytoplasm with TA-Ag-NPs (10 and $60 \mathrm{~nm}$ ), whether in NP or dissolved form, indicating that there may be a surface functionality dependence to their internalization. This agrees in principle with observations made by Malysheva et al., ${ }^{14}$ who suggested that internalized Ag was a strong indicator of Ag-NP toxicity, which in turn was dependent on the surface functionality due to a combination of factors including attachment to the cell. It should also be noted that Wang et al. used considerably different conditions, including a much higher concentration of $2000 \mu \mathrm{g} / \mathrm{L}$ compared to $40 \mu \mathrm{g} / \mathrm{L}$ $\mathrm{Ag}$ and a longer exposure time ( $24 v s 48 \mathrm{~h}$ ), so TA-Ag-NPs may also accumulate $\mathrm{Ag}$ inside algae under different conditions.

Finally, this study highlights the challenges associated with investigating three-dimensional problems with two-dimensional instrumental techniques. The fact that these images are either (a) images of reduced dimension due to relatively large depth of field or (b) thin sections (the section may not contain NPs) of a larger three-dimensional body makes the task of distinguishing extracellular $v s$ intracellular NPs challenging. One way to enable this differentiation is by tomographic methods, either by computationally reconstructing a series of projections acquired over a range of angles, by making a 3D stack from a series of thin sections, or by sequential imaging of sputtered surfaces by NanoSIMS. This has been demonstrated with various methods such as DF-LM, ${ }^{37}$ soft X-ray microscopy, ${ }^{44} \mathrm{TEM}^{45}$ and focused ion beams (FIBs), as well as with NanoSIMS, ${ }^{46}$ and combining the appropriate $2 \mathrm{D}$ technique with a 3D tomographic approach will provide further insight into bio-nano interactions. Coherent anti-Stokes Raman spectroscopy (CARS) imaging can also offer nondestructive analysis. ${ }^{19}$ Super-resolution microscopy is a promising development for these applications too, with resolution that can surpass the traditional limits and has been applied more recently to nanoparticlecontaining systems. ${ }^{47}$ While it is yet to be fully explored for bio-nano interactions, if combined with confocal imaging, this could offer a powerful alternative to in vacuo methods, since this can be performed under hydrated, atmospheric conditions.

\section{CONCLUSIONS}

We have demonstrated a complementary multimodal methodology for analyzing Ag-NPs interactions with the green algae 
R. subcapitata. The combination of optical, electron, and mass spectrometric imaging provides a powerful approach to examine the fate and associations of ENMs in biological systems, overcoming the individual limitations posed by each technique taken in isolation. The three techniques provide different levels of detail in the bio-nano interactions and together clearly revealed that the majority of the Ag-NPs or their dissolution products were localized around the algal cell walls. At the same time, SEM and NanoSIMS images of algal cells exposed to $10 \mathrm{~nm}$ bPEI-Ag-NPs suggested localized "hot-spots" for Ag or that some Ag-NPs entered and deposited inside the cells beyond the periplasmic space. The successful application of the methodology in future studies will enable advances in understanding Ag-NP-algae interactions and, more broadly, of bio-nano interactions using this combination of micro- and nanoscopic imaging techniques.

\section{MATERIALS AND METHODS}

Algal Culture and Exposure to Silver Nanoparticles. Raphidocelis subcapitata stock culture was purchased from SAG Culture Collection in Goettingen (Germany), strain 61.81, and kept as a continuous culture on site. Working cultures were subcultured from this stock and grown in standard OECD test medium. ${ }^{18}$ They were grown in $250 \mathrm{~mL}$ suspension culture flasks (Greiner Bio-One, Stonehouse, UK) in a controlled-temperature room $\left(22^{\circ} \mathrm{C}\right)$ under continuous fluorescent lighting (Agrobrite T12 fluorescence growth tubes, $54 \mathrm{~W}, 6400 \mathrm{~K}$ ) and stirred at $80 \mathrm{rpm}$ on an orbital shaker. The cell density was measured using a flow cytometer (BD Accuri C6, BD Biosciences). The cultures were conditioned for more than a week in the medium prior to exposing them to Ag-NPs.

Biopure silver nanoparticles were purchased from nanoComposix (San Diego, CA, USA) with two oppositely charged surface coatings: negatively charged tannic acid and positively charged branchedpolyethylenimine. Both types of Ag-NPs were purchased with two core sizes, 10 and $60 \mathrm{~nm}$, and were fully characterized by the manufacturer. All Ag-NPs were also recharacterized prior to the experiments using dynamic light scattering (DLS, Nicomp 380 ZLS, Particle Sizing Systems) and SEM (Quanta 450 FEG-ESEM, FEI Company) as described previously, ${ }^{48}$ and a summary of the major results is shown in Table 2.

R. subcapitata was allowed to grow for $48 \mathrm{~h}$ prior to being exposed to Ag-NPs during the midexponential phase of the growth profile and at a population greater than $3 \times 10^{5}$ cells $/ \mathrm{mL}$. Exposure was delayed in comparison to the OECD protocol ${ }^{18}$ in order to ensure that algal population remained sufficiently high after Ag-NPs were introduced (preliminary tests showed that the cell population was too low for the subsequent fixation and imaging processes to be practical if the OECD protocol was strictly followed). The cultures $(100 \mathrm{~mL}$ for each exposure) were exposed to Ag-NPs at two concentrations (15 and $40 \mu \mathrm{g} / \mathrm{L}$ ) where algae-NP interactions were expected, and the same Ag concentrations were employed for the two types of Ag-NPs so that where differences in their interactions were observed it may be directly attributable to the surface functionality. After $24 \mathrm{~h}$ of exposure, the cultures were separated from the media by centrifugation in $2 \times 50 \mathrm{~mL}$ tubes $(3660 \mathrm{~g}, 20 \mathrm{~min}$ ) and resuspended in $0.5 \mathrm{~mL}$ of fresh medium. Aliquots were taken at the start of the exposure period and were immediately acidified to $2 \% \mathrm{HNO}_{3}(\mathrm{v} / \mathrm{v})$ for analysis by ICP-MS
(Agilent 8800 Triple Quadrupole ICP-MS). As the measured concentrations were only slightly different from the nominal concentrations, they are referred to as 15 and $40 \mu \mathrm{g} / \mathrm{L}$ exposures for simplicity (Table 2). Additionally, as a comparison, cells were exposed to an $\mathrm{EC}_{50}$ concentration of $2 \mu \mathrm{g} / \mathrm{L} \mathrm{Ag}^{+}$ions in the form of $\mathrm{AgNO}_{3}$.

Sample Preparation for Electron Microscopy and NanoSIMS Analyses. The collected samples were chemically fixed using a mixture of $2 \%$ paraformaldehyde, $2.5 \%$ glutaraldehyde, and $0.1 \%$ tannic acid powder in a sodium cocodylate buffer $(0.1 \mathrm{M})$, using a microwaveassisted method. The cells were stained with $1 \% \mathrm{OsO}_{4}$ and with $2 \%$ uranyl acetate in order to give SEM contrast and rinsed with ultrapure water. The fixed cells were dehydrated incrementally with acetone (5 min each of six steps: $70,80,90$, and $3 \times 100 \%$ acetone) prior to stepwise infiltration of Spurr's resin in acetone at 1:3, 1:1, and 3:1 ratios for $15 \mathrm{~min}$ each. Finally, 100\% resin was infiltrated and incubated for $3 \times 15 \mathrm{~min}$ on a rocker and subsequently polymerized at $100{ }^{\circ} \mathrm{C}$. Sections of $0.5 \mu \mathrm{m}$ were sliced using a Reichert Ultracut E microtome for both SEM and NanoSIMS analysis. The sections were placed on a water droplet on a Si wafer and dried flat on a hot plate at $60{ }^{\circ} \mathrm{C}$.

SEM and NanoSIMS Imaging. SEM images of the fixed algae were acquired on two instruments: (1) a Carl Zeiss NVision 40 FIB with a field emission (FE) SEM (Gemini column) and (2) a Carl Zeiss Merlin FE SEM with a Gemini II column operating in high-resolution mode (Carl Zeiss, Oberkochen). Images were obtained using the in-column secondary electron ("InLens") and energy selective backscatter (ESB) detectors. The system was operated with accelerating voltages between 1 and $5 \mathrm{keV}$ (primarily at $2 \mathrm{keV}$ as per established protocol ${ }^{49}$ and a short working distance $(\mathrm{WD}, 3-7 \mathrm{~mm})$ to maximize resolution (smaller divergence) and detector efficiency (closer to the detector). SEM images were acquired directly from the sections: at these low voltages, sample charging was minimal for the majority of the samples even without the application of a conductive coating.

Nanoscale SIMS images were acquired with a NanoSIMS 50 ion microprobe (CAMECA, Gennevilliers, France) using the $\mathrm{Cs}^{+}$primary ion source operated at $16 \mathrm{keV}$. For NanoSIMS analysis, the samples were coated with $10 \mathrm{~nm}$ of platinum (Pt) to prevent charging. Prior to imaging, the region of interest was repeatedly scanned using a highcurrent ion beam to remove the $\mathrm{Pt}$ coating and implant $\mathrm{Cs}^{+}$ions to reach steady state and thereby improve secondary ion generation from the area. The implanted dose was $1 \times 10^{17} \mathrm{Cs}^{+}$ions $/ \mathrm{cm}^{2}$. The samples were imaged with the $300 \mu \mathrm{m}$ aperture and a current of 1.4-2 pA with a dwell time of $60 \mathrm{~ms}$. The NanoSIMS 50 is equipped with a magnetic sector analyzer with five secondary ion detectors. The first four detectors were positioned to collect ${ }^{12} \mathrm{C}^{14} \mathrm{~N}^{-}$(for carbon), ${ }^{32} \mathrm{~S}^{-}$and ${ }^{31} \mathrm{P}^{12} \mathrm{C}^{-}$ (for phosphorus), and ${ }^{107} \mathrm{Ag}^{-}$ions. The fifth detector was varied between ${ }^{98} \mathrm{Mo}^{16} \mathrm{O}^{-},{ }^{107} \mathrm{Ag}^{16} \mathrm{O}^{-}$, and ${ }^{107} \mathrm{Ag}^{32} \mathrm{~S}^{-}$depending on which signal was required: despite the high mass resolution $(M / \Delta M \approx 5000)$ of the NanoSIMS instrument, the ${ }^{107} \mathrm{Ag}^{-}$ion $(|m / z|=106.9051)$ could not be resolved from the ${ }^{95} \mathrm{Mo}^{12} \mathrm{C}^{-}$ion $(|m / z|=106.9058)$, and similarly ${ }^{109} \mathrm{Ag}^{-} v{ }^{97} \mathrm{Mo}^{12} \mathrm{C}^{-}$. Thus, ${ }^{98} \mathrm{Mo}^{16} \mathrm{O}^{-}(m / z=114)$ was used in the case of dissolved $\mathrm{Ag}$ exposure $\left(\mathrm{AgNO}_{3}\right)$ to image its distribution independently of $\mathrm{Ag}$, as a means to cross-examine whether any Mo accumulation could be observed and whether it correlated to that of Ag. Secondary electrons are also generated during the sputtering process and were collected from the same region of interest to show morphology and topography. All images were processed using the OpenMIMS plugin (Harvard, Cambridge, MA, USA) within the ImageJ software and are presented on an arbitrary linear scale with blue indicating regions of low intensity, while red indicates regions of high intensity.

Table 2. Size (by TEM, SEM, and DLS), $\zeta$-Potential ( $\zeta$-V), and the Concentrations of Ag-NPs Used for Exposure to R. subcapitata

$\begin{array}{lrccccc} & \mathrm{TEM}^{a}(\mathrm{~nm}) & \text { SEM }(\mathrm{nm}) & \text { DLS }(\mathrm{nm}) & \zeta \text {-V }(\mathrm{mV}) & \text { low }(\mu \mathrm{g} / \mathrm{L}) & \text { high }(\mu \mathrm{g} / \mathrm{L}) \\ \text { TA-Ag-NPs, } 10 \mathrm{~nm} & 10.0 \pm 1.8 & & 12.4 \pm 0.7 & -22.1 & 14.4 & 37.8 \\ \text { TA-Ag-NPs, } 60 \mathrm{~nm} & 60.8 \pm 6.6 & 49.7 \pm 7.7 & 56.3 \pm 1.0 & -33.1 & 16.4 & 39.5 \\ \text { bPEI-Ag-NPs, } 10 \mathrm{~nm} & 8.8 \pm 2.2 & & 15.8 \pm 2.3 & +20.6 & 14.7 & 41.6 \\ \text { bPEI-Ag-NPs, } 60 \mathrm{~nm} & 60.8 \pm 6.6 & 55.8 \pm 7.1 & 76.8 \pm 2.4 & +34.4 & 17.4 & 46.4\end{array}$

${ }^{a_{\text {TEM }}}$ sizes are as provided by the manufacturer, $\mathrm{TA}=$ tannic acid, $\mathrm{bPEI}=$ branched polyethylenimine, low and high indicate the two exposure concentrations used. 
Dark-Field Light Microscopy and Hyperspectral Imaging. Algal cultures for dark-field imaging were grown separately from a stock culture of R. subcapitata at the University of South Australia (acquired from the Australian National Algae Culture Collection), but with equivalent exposure ( $40 \mu \mathrm{g} / \mathrm{L}$ nominal) and under OECD testing conditions. After exposure to Ag-NPs, $R$. subcapitata were collected by filtration onto $0.8 \mu \mathrm{m}$ pore polycarbonate filters to remove all noninteracting Ag-NPs and were resuspended in fresh OECD media for imaging by enhanced dark-field light microscopy. Drops of the suspensions were then placed on microscope slides and examined under a coverslip with the Cytoviva enhanced dark-field microscope with $60 \times$ oil immersion objective. Samples without filtration were also analyzed for comparison, and while the background was slightly brighter with some NPs visible, there were no significant differences in the algae and the NP interactions did not appear to be affected. Hyperspectral data $(400-1000 \mathrm{~nm})$ were collected from selected cells that had particularly bright areas of scattering, and the data were extracted using MATLAB.

\section{ASSOCIATED CONTENT}

\section{S Supporting Information}

The Supporting Information is available free of charge on the ACS Publications website at DOI: 10.1021/acsnano.7b04556.

Additional figures (PDF)

\section{AUTHOR INFORMATION}

\section{Corresponding Author}

*Tel: +44-14916-92633. E-mail: ryosek@ceh.ac.uk (R. Sekine). ORCID

Ryo Sekine: 0000-0001-9980-0603

\section{Author Contributions}

${ }^{\#}$ R. Sekine and K. L. Moore equally contributed to the work described in this article.

\section{Notes}

The authors declare no competing financial interest.

Research data underlying this publication is also available from the Mendeley Data repository at http://dx.doi.org/10.17632/ mc84myxghz.1

\section{ACKNOWLEDGMENTS}

The authors thank the Australian Nanotechnology Network (Overseas Travel Fellowship) and the University of South Australia (Early Career International Travel Award) for funding the visit of R.S. that enabled this collaboration. The funding support from the Horizon 2020 Marie Sklodowska-Curie Actions Individual Fellowship (MolNANOtox) and the Australian Research Council (ARC) Discovery Project DP120101115 are also gratefully acknowledged. K.L.M. was supported by the Engineering and Physical Sciences Research Council Fellowship EP/I026584/1. The time of M.M. and C.S. were supported by the European projects GUIDEnano (CP-FP7 604387) and NanoFASE (H2020-646002). E.D. and E.L. were supported by ARC Future Fellowships FT130101003 and FT100100337, respectively. We would also like to thank L. Hughes from Oxford Brookes University for her assistance in sample fixation and embedding, and the University of Manchester for funding to make this paper open access.

\section{REFERENCES}

(1) Forbrugerrådet Tænk, The Nanodatabase. http://nanodb.dk/en/ (accessed 07/18/2016).

(2) Woodrow Wilson Institute, The Project on Emerging Nanotechnologies. http://www.nanotechproject.org/ (accessed 07/18/ 2016).
(3) Schaumann, G. E.; Philippe, A.; Bundschuh, M.; Metreveli, G.; Klitzke, S.; Rakcheev, D.; Grun, A.; Kumahor, S. K.; Kuhn, M.; Baumann, T.; et al. Understanding the Fate and Biological Effects of Agand $\mathrm{TiO}_{2}$-Nanoparticles in the Environment: The Quest for Advanced Analytics and Interdisciplinary Concepts. Sci. Total Environ. 2015, 535, $3-19$.

(4) Schultz, C.; Powell, K.; Crossley, A.; Jurkschat, K.; Kille, P.; Morgan, A. J.; Read, D.; Tyne, W.; Lahive, E.; Svendsen, C.; et al. Analytical Approaches to Support Current Understanding of Exposure, Uptake and Distributions of Engineered Nanoparticles by Aquatic and Terrestrial Organisms. Ecotoxicology 2015, 24, 239-261.

(5) Gottschalk, F.; Nowack, B. The Release of Engineered Nanomaterials to the Environment. J. Environ. Monit. 2011, 13, 1145-1155.

(6) Sun, T. Y.; Gottschalk, F.; Hungerbühler, K.; Nowack, B. Comprehensive Probabilistic Modelling of Environmental Emissions of Engineered Nanomaterials. Environ. Pollut. 2014, 185, 69-76.

(7) Commission Delegated Regulation (EU) No 1363/2013 of 12 December 2013 Amending Regulation (EU) No 1169/2011 of the European Parliament and of the Council on the Provision of Food Information to Consumers as Regards the Definition of 'Engineered Nanomaterials'. Official J. European Union 2013, L343, 26-28.

(8) McGregor, J. E.; Staniewicz, L. T. L.; Guthrie, S. E.; Donald, A. M. Environmental Scanning Electron Microscopy in Cell Biology. In Cell Imaging Techniques: Methods and Protocols; Taatjes, D. J.; Roth, J., Eds.; Humana Press: Totowa, NJ, 2013; pp 493-516.

(9) Danilatos, G. D. Review and Outline of Environmental SEM at Present. J. Microsc. (Oxford, U. K.) 1991, 162, 391-402.

(10) Thompson, R. F.; Walker, M.; Siebert, C. A.; Muench, S. P.; Ranson, N. A. An Introduction to Sample Preparation and Imaging by Cryo-Electron Microscopy for Structural Biology. Methods (Amsterdam, Neth.) 2016, 100, 3-15.

(11) Nazaretski, E.; Lauer, K.; Yan, H.; Bouet, N.; Zhou, J.; Conley, R.; Huang, X.; Xu, W.; Lu, M.; Gofron, K.; et al. Pushing the Limits: An Instrument for Hard X-Ray Imaging Below $20 \mathrm{~nm}$. J. Synchrotron Radiat. 2015, 22, 336-341.

(12) Robinson, I.; Yang, Y.; Zhang, F.; Lynch, C.; Yusuf, M.; Cloetens, P. Nuclear Incorporation of Iron During the Eukaryotic Cell Cycle. J. Synchrotron Radiat. 2016, 23, 1490-1497.

(13) Kyriacou, B.; Moore, K. L.; Paterson, D.; de Jonge, M. D.; Howard, D. L.; Stangoulis, J.; Tester, M.; Lombi, E.; Johnson, A. A. T. Localization of Iron in Rice Grain Using Synchrotron X-Ray Fluorescence Microscopy and High Resolution Secondary Ion Mass Spectrometry. J. Cereal Sci. 2014, 59, 173-180.

(14) Malysheva, A.; Voelcker, N.; Holm, P. E.; Lombi, E. Unraveling the Complex Behavior of Ag-NPs Driving NP-Cell Interactions and Toxicity to Algal Cells. Environ. Sci. Technol. 2016, 50, 12455-12463.

(15) Ivask, A.; Kurvet, I.; Kasemets, K.; Blinova, I.; Aruoja, V.; Suppi, S.; Vija, H.; Käkinen, A.; Titma, T.; Heinlaan.; et al. Size-Dependent Toxicity of Silver Nanoparticles to Bacteria, Yeast, Algae, Crustaceans and Mammalian Cells. PLoS One 2014, 9, e102108.

(16) Navarro, E.; Baun, A.; Behra, R.; Hartmann, N. B.; Filser, J.; Miao, A.-j.; Quigg, A.; Santschi, P. H.; Sigg, L. Environmental Behavior and Ecotoxicity of Engineered Nanoparticles to Algae, Plants, and Fungi. Ecotoxicology 2008, 17, 372-386.

(17) Navarro, E.; Piccapietra, F.; Wagner, B.; Marconi, F.; Kaegi, R.; Odzak, N.; Sigg, L.; Behra, R. Toxicity of Silver Nanoparticles to Chlamydomonas reinhardtii. Environ. Sci. Technol. 2008, 42, 8959-8964.

(18) The Organisation for Economic Co-operation and Development, OECD Guidelines for the Testing of Chemicals. In Guideline 201: Freshwater Alga and Cyanobacteria, Growth Inhibition Test, 2002, Rev. 2006.

(19) Ribeiro, F.; Gallego-Urrea, J. A.; Goodhead, R. M.; Van Gestel, C. A.; Moger, J.; Soares, A. M.; Loureiro, S. Uptake and Elimination Kinetics of Silver Nanoparticles and Silver Nitrate by Raphidocelis subcapitata: The Influence of Silver Behaviour in Solution. Nanotoxicology 2015, 9, 686-695.

(20) Sorensen, S. N.; Baun, A. Controlling Silver Nanoparticle Exposure in Algal Toxicity Testing - a Matter of Timing. Nanotoxicology 2015, 9, 1-9.10.3109/17435390.2014.913728 
(21) Ben-Sasson, M.; Zodrow, K. R.; Genggeng, Q.; Kang, Y.; Giannelis, E. P.; Elimelech, M. Surface Functionalization of Thin-Film Composite Membranes with Copper Nanoparticles for Antimicrobial Surface Properties. Environ. Sci. Technol. 2013, 48, 384-393.

(22) Ivask, A.; Visnapuu, M.; Vallotton, P.; Marzouk, E. R.; Lombi, E.; Voelcker, N. H. Quantitative Multimodal Analyses of Silver Nanoparticle-Cell Interactions: Implications for Cytotoxicity. NanoImpact 2016, 1, 29-38.

(23) Mortimer, M.; Gogos, A.; Bartolomé, N.; Kahru, A.; Bucheli, T. D.; Slaveykova, V. I. Potential of Hyperspectral Imaging Microscopy for Semi-Quantitative Analysis of Nanoparticle Uptake by Protozoa. Environ. Sci. Technol. 2014, 48, 8760-8767.

(24) Yang, X.; Jiang, C.; Hsu-Kim, H.; Badireddy, A. R.; Dykstra, M.; Wiesner, M.; Hinton, D. E.; Meyer, J. N. Silver Nanoparticle Behavior, Uptake, and Toxicity in Caenorhabditis elegans: Effects of Natural Organic Matter. Environ. Sci. Technol. 2014, 48, 3486-3495.

(25) Liebl, H. Ion Probe Microanalysis. J. Phys. E: Sci. Instrum. 1975, 8, 797-808.

(26) Herrmann, A. M.; Ritz, K.; Nunan, N.; Clode, P. L.; Pett-Ridge, J.; Kilburn, M. R.; Murphy, D. V.; O’Donnell, A. G.; Stockdale, E. A. NanoScale Secondary Ion Mass Spectrometry - a New Analytical Tool in Biogeochemistry and Soil Ecology: A Review Article. Soil Biol. Biochem. 2007, 39, 1835-1850.

(27) Eichorst, S. A.; Strasser, F.; Woyke, T.; Schintlmeister, A.; Wagner, M.; Woebken, D. Advancements in the Application of NanoSIMS and Raman Microspectroscopy to Investigate the Activity of Microbial Cells in Soils. FEMS Microbiol. Ecol. 2015, 91, fiv106.

(28) Mueller, C. W.; Kölbl, A.; Hoeschen, C.; Hillion, F.; Heister, K.; Herrmann, A. M.; Kögel-Knabner, I. Submicron Scale Imaging of Soil Organic Matter Dynamics Using NanoSIMS - from Single Particles to Intact Aggregates. Org. Geochem. 2012, 42, 1476-1488.

(29) Kopittke, P. M.; Moore, K. L.; Lombi, E.; Gianoncelli, A.; Ferguson, B. J.; Blamey, F. P. C.; Menzies, N. W.; Nicholson, T. M.; McKenna, B. A.; Wang, P.; et al. Identification of the Primary Lesion of Toxic Aluminum in Plant Roots. Plant Physiol. 2015, 167, 1402-1411.

(30) Moore, K. L.; Schroder, M.; Lombi, E.; Zhao, F. J.; McGrath, S. P.; Hawkesford, M. J.; Shewry, P. R.; Grovenor, C. R. M. NanoSIMS Analysis of Arsenic and Selenium in Cereal Grain. New Phytol. 2010, 185, 434-445.

(31) Georgantzopoulou, A.; Balachandran, Y. L.; Rosenkranz, P.; Dusinska, M.; Lankoff, A.; Wojewodzka, M.; Kruszewski, M.; Guignard, C.; Audinot, J.-N.; Girija, S.; et al. Ag Nanoparticles: Size- and SurfaceDependent Effects on Model Aquatic Organisms and Uptake Evaluation with NanoSIMS. Nanotoxicology 2013, 7, 1168-1178.

(32) Wang, S.; Lv, J.; Ma, J.; Zhang, S. Cellular Internalization and Intracellular Biotransformation of Silver Nanoparticles in Chlamydomonas reinhardtii. Nanotoxicology 2016, 10, 1129-35.

(33) El Badawy, A. M.; Silva, R. G.; Morris, B.; Scheckel, K. G.; Suidan, M. T.; Tolaymat, T. M. Surface Charge-Dependent Toxicity of Silver Nanoparticles. Environ. Sci. Technol. 2010, 45, 283-287.

(34) Badireddy, A. R.; Wiesner, M. R.; Liu, J. Detection, Characterization, and Abundance of Engineered Nanoparticles in Complex Waters by Hyperspectral Imagery with Enhanced Darkfield Microscopy. Environ. Sci. Technol. 2012, 46, 10081-10088.

(35) Theoret, T.; Wilkinson, K. J. Evaluation of Enhanced Darkfield Microscopy and Hyperspectral Analysis to Analyse the Fate of Silver Nanoparticles in Wastewaters. Anal. Methods 2017, 9, 3920-3928.

(36) Mogensen, K. B.; Kneipp, K. Size-Dependent Shifts of Plasmon Resonance in Silver Nanoparticle Films Using Controlled Dissolution: Monitoring the Onset of Surface Screening Effects. J. Phys. Chem. C 2014, 118, 28075-28083.

(37) Vallotton, P.; Angel, B.; McCall, M.; Osmond, M.; Kirby, J. Imaging Nanoparticle-Algae Interactions in Three Dimensions Using Cytoviva Microscopy. J. Microsc. (Oxford, U. K.) 2015, 257, 166-169.

(38) Machado, M. D.; Soares, E. V. Modification of Cell Volume and Proliferative Capacity of Pseudokirchneriella subcapitata Cells Exposed to Metal Stress. Aquat. Toxicol. 2014, 147, 1-6.

(39) Yamagishi, T.; Yamaguchi, H.; Suzuki, S.; Horie, Y.; Tatarazako, N. Cell Reproductive Patterns in the Green Alga Pseudokirchneriella subcapitata (=Selenastrum capricornutum) and Their Variations under Exposure to the Typical Toxicants Potassium Dichromate and 3,5-DCP. PLoS One 2017, 12, e0171259.

(40) Garrett, D. C. Effects of Methanol, Atrazine, and Copper on the Ultrastructure of Pseudokirchneriella subcapitata (Selenastrum capricornutum); University of North Texas: Denton, TX, 2004.

(41) Geitner, N. K.; Marinakos, S. M.; Guo, C.; O’Brien, N.; Wiesner, M. R. Nanoparticle Surface Affinity as a Predictor of Trophic Transfer. Environ. Sci. Technol. 2016, 50, 6663-6669.

(42) Li, X.; Schirmer, K.; Bernard, L.; Sigg, L.; Pillai, S.; Behra, R. Silver Nanoparticle Toxicity and Association with the Alga Euglena gracilis. Environ. Sci.: Nano 2015, 2, 594-602.

(43) Leonardo, T.; Farhi, E.; Pouget, S.; Motellier, S.; Boisson, A.-M.; Banerjee, D.; Rébeillé, F.; den Auwer, C.; Rivasseau, C. Silver Accumulation in the Green Microalga Coccomyxa actinabiotis: Toxicity, In Situ Speciation, and Localization Investigated Using Synchrotron XAS, XRD, and TEM. Environ. Sci. Technol. 2016, 50, 359-367.

(44) Drescher, D.; Guttmann, P.; Buchner, T.; Werner, S.; Laube, G.; Hornemann, A.; Tarek, B.; Schneider, G.; Kneipp, J. Specific Biomolecule Corona Is Associated with Ring-Shaped Organization of Silver Nanoparticles in Cells. Nanoscale 2013, 5, 9193-9198.

(45) de Jonge, N.; Sougrat, R.; Northan, B. M.; Pennycook, S. J. ThreeDimensional Scanning Transmission Electron Microscopy of Biological Specimens. Microsc. Microanal. 2010, 16, 54-63.

(46) Jiang, H.; Passarelli, M. K.; Munro, P. M. G.; Kilburn, M. R.; West, A.; Dollery, C. T.; Gilmore, I. S.; Rakowska, P. D. High-Resolution SubCellular Imaging by Correlative NanoSIMS and Electron Microscopy of Amiodarone Internalisation by Lung Macrophages as Evidence for Drug-induced Phospholipidosis. Chem. Commun. 2017, 53, 1506-1509.

(47) van der Zwaag, D.; Vanparijs, N.; Wijnands, S.; De Rycke, R.; De Geest, B. G.; Albertazzi, L. Super Resolution Imaging of Nanoparticles Cellular Uptake and Trafficking. ACS Appl. Mater. Interfaces 2016, 8, 6391-6399.

(48) Sekine, R.; Khurana, K.; Vasilev, K.; Lombi, E.; Donner, E. Quantifying the Adsorption of Ionic Silver and Functionalized Nanoparticles During Ecotoxicity Testing: Test Container Effects and Recommendations. Nanotoxicology 2015, 9, 1005-1012.

(49) Jiang, H.; Favaro, E.; Goulbourne, C. N.; Rakowska, P. D.; Hughes, G. M.; Ryadnov, M. G.; Fong, L. G.; Young, S. G.; Ferguson, D. J. P.; Harris, A. L.; et al. Stable Isotope Imaging of Biological Samples with High Resolution Secondary Ion Mass Spectrometry and Complementary Techniques. Methods (Amsterdam, Neth.) 2014, 68, 317-324. 